\title{
Contribution of dendritic cell/T cell interactions to triggering and maintaining autoimmunity
}

\author{
Carolina Llanos ${ }^{1,2}$, Leandro J. Carreño ${ }^{1}$ and Alexis M. Kalergis ${ }^{1,2, *}$ \\ ' Millennium Nucleus on Immunology and Immunotherapy, Departamento de Genética Molecular y Microbiología, Facultad de Ciencias Biológicas Pontificia Universidad Católica. \\ 2 Departamento de Reumatología, Facultad de Medicina, Pontificia Universidad Católica de Chile.
}

\begin{abstract}
Under healthy conditions, there is a balance between tolerance to self-tissue constituents and immunity against foreign antigens. Autoimmunity diseases (AD) take place when that equilibrium is disrupted and the immune response is directed to self-antigens, leading to injury or destruction of host tissues. The mechanisms conducing to the loss of immune tolerance remain largely unknown. The recent appearance of biological therapies has contributed to significant reduction in morbidity. However, currently available therapies are associated with important side effects and work only as palliative treatments. Dendritic cells (DCs) have emerged as key players in developing and maintaining adaptive immunity due to their capacity to prime and modulate T cell function. Therefore, because DCs work as central modulators of immune tolerance, it is likely that alterations in their function can lead to the onset of autoimmune-inflammatory diseases. By modulating DC function, novel pathways in antigen-specific tolerance could be established. In this article, the possible contribution of altered DC-T cell interactions to the onset of autoimmunity are discussed. In addition, we expand on the notion that some of the functions of these cells could be relevant targets for intervening therapies aimed to restore the balance or even prevent the loss of tolerance.
\end{abstract}

Key words: dendritic cells; T cells; immunological synapse; autoimmunity; tolerance.

\section{INTRODUCTION}

Autoimmunity takes place when the immune system recognizes and attacks host tissues on a chronic manner leading to organ damage and disease. Autoimmune disorders include a wide variety of distinctive clinical entities that can share common pathways of pathogenesis leading to damage and potential failure of different organs. They most predominantly affect women, many of them in childbearing age. Although individually they are rare disorders, their prevalence is increasing in the world and it is estimated to reach approximately 5 to $8 \%$ in the US [http:/ / www3.niaid.nih.gov/ topics/autoimmune/]. These syndromes have unclear etiology and include well known diseases such as Rheumatoid Arthritis (RA), an incurable disorder characterized by the presence of $\mathrm{CD}^{+} \mathrm{T}$ cells infiltrating the synovial membrane of diarthrodial joints and by the production of strong pro-inflammatory cytokines, such as TNF- $\alpha$, Interleukin (IL) and Interleukin-6 by macrophages and fibroblasts also present at the synovium. Untreated inflammation can lead to severe cartilage and bone destruction (Choy and Panayi 2001). Another example of tissuespecific autoimmune disease is Type 1 Diabetes (t-1D), which involves T cell- and antibody-mediated inflammation of the islets with failure and death of pancreatic $\beta$-cells conducting to severe hyperglicemia due to insulin deficiency (Eizirik, Colli et al. 2009). On the other hand, examples of multisystemic autoimmune disorders include Systemic Lupus Eryhematosus (SLE), an autoimoune disorder chorocterized forthes hyperproduction of auto-antibodies against double-stranded DNA, nucleosomes, ribonucleoproteins and other nuclear antigens that cause deposit of immune complexes in blood vessels with macrophage and complement activation, inflammation and tissue damage
(Rahman and Isenberg 2008) another example is Multiple Sclerosis (MS) chracterized by the presence of auto-reactive $\mathrm{T}$ cells against myelin causing demyelination, axonal damage and neuronal loss (Wingerchuk, Lucchinetti et al. 2001).

Although substantial progress has been made in the treatment and prevention of both specific and systemic autoimmune disorders, therapeutic options remain limited. In the case of RA, while the emergence of TNF- $\alpha$ blockade has stalled the progression of joint damage and achieved low disease activity and disease remission in a high proportion of studies, agents can lead to opportunistic infections. In addition, anti-TNF antibodies are expensive medications and several patients have shown that remission and satisfactory responses are not sustained over time (Taylor and Feldmann 2009). The current approach for t-1D consists of replacement therapy involving insulin analogs, unfortunately immunomodulating strategies aimed at inducing remission, such as anti-CD3 antibody, DiaPep277 and GAD65 have shown only reduced efficacy (Bresson and von Herrath 2007; Waldron-Lynch and Herold 2009). No new agents for SLE treatment have been approved by the FDA in the past 30 years and current treatment is based on unspecific immunosuppressive drugs, such as cyclophosphamide, mycophenolate mofetil and steroids, which are only partially effective and can contribute to promoting opportunistic infections in patients (Dall'Era and Wofsy 2009; Eisenberg 2009). In addition, initial small uncontrolled trials using Rituximab, a chimeric anti-CD20 monoclonal antibody, showed promising results in SLE (Leandro, Edwards et al. 2002; Smith, Jones et al. 2006; Tokunaga, Saito et al. 2007). However, recent results from a randomized double blind study showed that CD20 depletion therapy is not effective in patients with active

\footnotetext{
* Address correspondence and reprint requests to A. M. Kalergis, Millennium Nucleus on Immunology and Immunotherapy, Departamento de Genética Molecular y Microbiología, Facultad de Ciencias Biológicas, Pontificia Universidad Católica de Chile. Portugal \#49, Santiago, Chile. Phone: 56-2-686-2842, Fax: 56-2-686-2185, E-mail address: akalergis@bio.puc.cl and kalergis@vtr.net
} 
extra-renal SLE (Merrill, Neuwelt et al.). On the other hand, current available therapies for MS include formulations containing interferon- $\beta$, glatiramer acetate, mitoxantrone and natalizumab, a monoclonal antibody against $\alpha 4$-integrin. All these individual treatments have shown to improve disease activity, but they have an unclear impact on disease progression and can have significant side effects (DeAngelis and Lublin 2008). Therefore, given that no curative effective therapies are currently available for any of these ailments and the burden of disease for patients is increasing steadily, significant research efforts have been invested on elucidating the pathogenic mechanisms leading to these disorders. The understanding of such mechanisms will contribute to finding novel and safer therapies for autoimmune-inflammatory diseases.

During the past few years, significant amount of empirical data has been generated to support the notion that DCs work as professional antigen-presenting cells required for regulating the nature of the immune response of the host. Depending on their functional status, DCs can either prevent or promote adaptive immunity in an antigen specific fashion (Lanzavecchia and Sallusto 2001). During infection, DCs protect the host by priming naïve $\mathrm{T}$ cells that can specifically recognize pathogen-derived antigens (Brimnes, Bonifaz et al. 2003). Conversely, in the absence of infection or during steadystate conditions, DCs contribute to maintaining central and peripheral tolerance to self-antigens, by either inducing anergy of auto-reactive $\mathrm{T}$ cells or by stimulating the proliferation of regulatory T cells (Steinman, Hawiger et al. 2003; Yamazaki, Iyoda et al. 2003). When the fragile balance between tolerance and immunity is disrupted, DCs can promote and perpetuate the activation of auto-reactive $\mathrm{T}$ cells leading to autoimmunity. Because $\mathrm{T}$ cells are the main effectors of adaptive immunity and DCs have been shown to be essential for initiating and controlling $\mathrm{T}$ cell function, the present review will bring out the particular role of each cell type during the loss of immune tolerance. In addition, recent findings will be discussed relative to the involvement of DC-T cell interactions on the modulation of $\mathrm{T}$ cell function and on determining the perpetuation of an autoimmune response

Modulation of central tolerance during adaptive immunity by DC-T cell interactions

$\mathrm{T}$ cell tolerance to self-antigens can be controlled at several points. Most self-reactive $\mathrm{T}$ cells are thought to be deleted in the thymus in a process known as central tolerance. Throughout development, the autoimmune regulator (AIRE) induces the expression of tissue specific antigens (TSA) in thymic epithelial cells (Tecs) (Peterson, Org et al. 2008). It has been shown that Tecs from the medulla are capable of presenting antigens to immature $\mathrm{CD} 4^{+} / \mathrm{CD}^{+}$double positive (DP) thymocytes to either enable their commitment to mature naive $\mathrm{T}$ cells (positive selection) or their deletion (negative selection) (Gallegos and Bevan 2004; Peterson, Org et al. 2008). This process is performed in collaboration with bone marrowderived DCs that are able to capture antigens from medullar Tecs and present them on MHC molecules to DP thymocytes (Gallegos and Bevan 2004). Nonetheless, recent data suggests that this process might not take place in the medulla, but rather at the thymic cortex, where DCs would induce clonal deletion of self-reactive thymocytes (McCaughtry, Baldwin et al.
2008). The outcome of a specific T cell clone depends on how it interacts with a self-peptide loaded in the MHC molecule (pMHC). During negative selection, thymocytes bearing TCRs that bind with high-affinity to pMHC molecules containing peptides from TSA are deleted or inactivated (Pircher, Rohrer et al. 1991; Starr, Jameson et al. 2003). As a result of this process, a peripheral self-reactive $\mathrm{T}$ cell repertoire is generated, composed of low and intermediate TCR self pMHC affinity clones, in opposition to a foreign pMHC-reactive clone pool assembled with the whole range of TCR affinities. An additional central mechanism to maintaining self-tolerance is the production of regulatory $\mathrm{T}$ cells (Tregs), called natural $\mathrm{T}$ regs. Interestingly, high affinity interactions between the TCR and self-pMHC have been shown to promote the generation and expansion of Tregs (Bettini and Vignali; Iruretagoyena, Wiesendanger et al. 2006).

As mentioned above, it is normal that some auto-reactive $\mathrm{T}$ cell clones are not deleted in the thymus and are released into circulation. However, in the periphery these $\mathrm{T}$ cells are rendered inactive by means of regulatory mechanisms that prevent activation of potentially pathogenic self-reactive $\mathrm{T}$ cell clones that could drive an adaptive immune response against self-tissues with the subsequent appearance of autoimmunity. Along these lines, it has been shown that AIRE can drive the expression of self-antigens by peripheral cells, which can in turn contribute to the deletion or suppression of $\mathrm{T}$ cells recognizing those self-antigens. It was recently shown by twophoton microscopy that extrathymic AIRE expressing cells are able to establish stable antigen-specific interactions with naïve auto-reactive $\mathrm{T}$ cells. These interactions are thought to prevent activation of these self-reactive $\mathrm{T}$ cells and consequently reinforce peripheral tolerance by means of the inhibition/ deletion of autoreactive $\mathrm{T}$ cells that could have escaped negative selection (Gardner, Devoss et al. 2008). In addition, other peripheral mechanisms mediated by DCs can also contribute to tolerance, including induction of T cell anergy, deletion or alternatively induction of antigen-specific $\mathrm{T}$ regs (Lutz and Kurts 2009).

Molecular interactions at the DC-T cell interface that contribute to the balance between tolerance and autoimmunity

DCs are the most efficient APCs at activating antigen-specific $\mathrm{T}$ cells (Banchereau and Steinman 1998). Because they are ubiquitously distributed in most tissues antigen capture is facilitated and capable of migrating lymphoid organs to encounter and subsequently activate antigen-specific $\mathrm{T}$ cells. In addition, they are able to process antigens and express large amounts of antigen presenting molecules, such as MHC-I, MHC-II and CD1 together with co-stimulatory molecules including CD80-CD86/B7.1-B7.2 (Banchereau and Steinman 1998). DCs are capable of sensing pathogen associated molecular patterns (PAMPs) through specific surface receptors termed toll-like receptors (TLR) and activate naïve $\mathrm{T}$ cells by upregulating the surface expression of both signal 1 (peptideMHC complexes) and signal 2 (co-stimulatory molecules) (Tobar, Carreno et al. 2006). A third signal, composed of cytokines, can contribute to determining the nature of the effector $\mathrm{T}$ cell response. Additionally, DCs contribute to the balance between immunity and tolerance by their ability to integrate in vivo activating and inhibitory signals triggered by pairs of activating/inhibitory receptors expressed on their 
surface. Example of these type of receptors are Fc $\gamma$ Rs, which recognize the Fc-fragment of $\mathrm{IgG}$ antibodies and consist of one inhibitory (FCR $\gamma \mathrm{IIB})$ and either three or four different activating receptors in mice (Fc $\gamma$ RI, III, IV) and humans (FcrRIA, IIA, IIIA and IIIB on neutrophils respectively). While the activating receptors signal through immunoreceptor tyrosine-based activating motifs or ITAM, inhibitory receptors do so through tyrosine-based inhibitory motifs or ITIM (Kalergis 2003; Iruretagoyena, Wiesendanger et al. 2006; Herrada, Contreras et al. 2007). Noteworthy, selective engagement of activating $\mathrm{Fc} \gamma$ receptors in bone-marrow DCs derived from $\mathrm{Fc}_{\mathrm{C}} \mathrm{RIIB}{ }^{-/}$mice results in enhanced DC maturation and induces tumor immunity (Kalergis and Ravetch 2002). Consistently, an Fc $\gamma$ RIIB deficiency can lead to overwhelming inflammation as evidenced by the increased susceptibility to EAE shown by Fc $\gamma$ RIIB knockout mice (Kalergis and Ravetch 2002; Iruretagoyena, Riedel et al. 2008). Furthermore, it has been recently shown that DCs from RA patients with inactive disease express higher levels of Fc $\gamma$ RIIB than active RA patients and healthy controls and that they inhibit TLR4 signaling, providing a novel mechanism as to how FC $\gamma$ RIIB exerts a regulatory role in immune responses (Wenink, Santegoets et al. 2009).

Depending on the signals provided by pMHC-presenting $\mathrm{DCs}, \mathrm{CD} 4^{+} \mathrm{T}$ cells can differentiate into at least 3 distinctive types of $T_{\text {helper }}\left(T_{h}\right)$ cells that secrete different cytokines in response to antigenic stimulation (Figure 1 ). $T_{h} 1$ cells produce interferon (IFN) $-\gamma$ and promote the induction of a cellular immune response with the subsequent activation of cytotoxic $\mathrm{T}$ lymphocytes (CTL) that are able to kill target cells. In addition, $\mathrm{T}_{\mathrm{h}} 1$ cells induce B cells to differentiate in IgG2a secreting cells in mice and $\operatorname{IgM}, \operatorname{Ig} A, \operatorname{IgG}_{1}, \operatorname{IgG}_{2}$ and $\operatorname{IgG}_{3}$ secreting cells in humans, increasing the release of antigen-specific antibodies of such isotypes. On the other hand, $\mathrm{T}_{\mathrm{h}} 2$ cells are characterized by the production of (IL)-4, IL-5 and IL-13, which are critical for inducing $B$ cell isotype switching into $\operatorname{IgG}_{1}$ and $\operatorname{IgE}$ in mice and $\mathrm{IgM}, \mathrm{IgG}_{4}$ and $\mathrm{IgE}$ in humans and the production of allergic-like immunity. The $T_{h} 1-T_{h} 2$ hypothesis was proposed as an attempt to find out whether different types of $\mathrm{CD} 4^{+} \mathrm{T}_{\mathrm{h}}$ cells were mediating the class of antibodies produced by $\mathrm{B}$ cells (Coffman 2006). Indeed, $T_{h} 1$ cells were initially thought to be involved in EAE and collagen-induced arthritis, until the unexpected observation that mice deficient in IFN- $\gamma$ or its receptor, a classic Th1 cytokine, were significantly more susceptible to developing EAE, instead of being protected from the disease (Krakowski and Owens 1996; Willenborg, Fordham et al. 1996). Along these lines, a recently described third subset of $T_{h}$ cells that secretes IL-17 is thought to be involved in chronic inflammation and tissue injury (Herrada, Contreras et al.; Diebold 2008). The case of $\mathrm{T}_{\mathrm{h}} 17$ responses is more complex because these cells not only are derived from a different

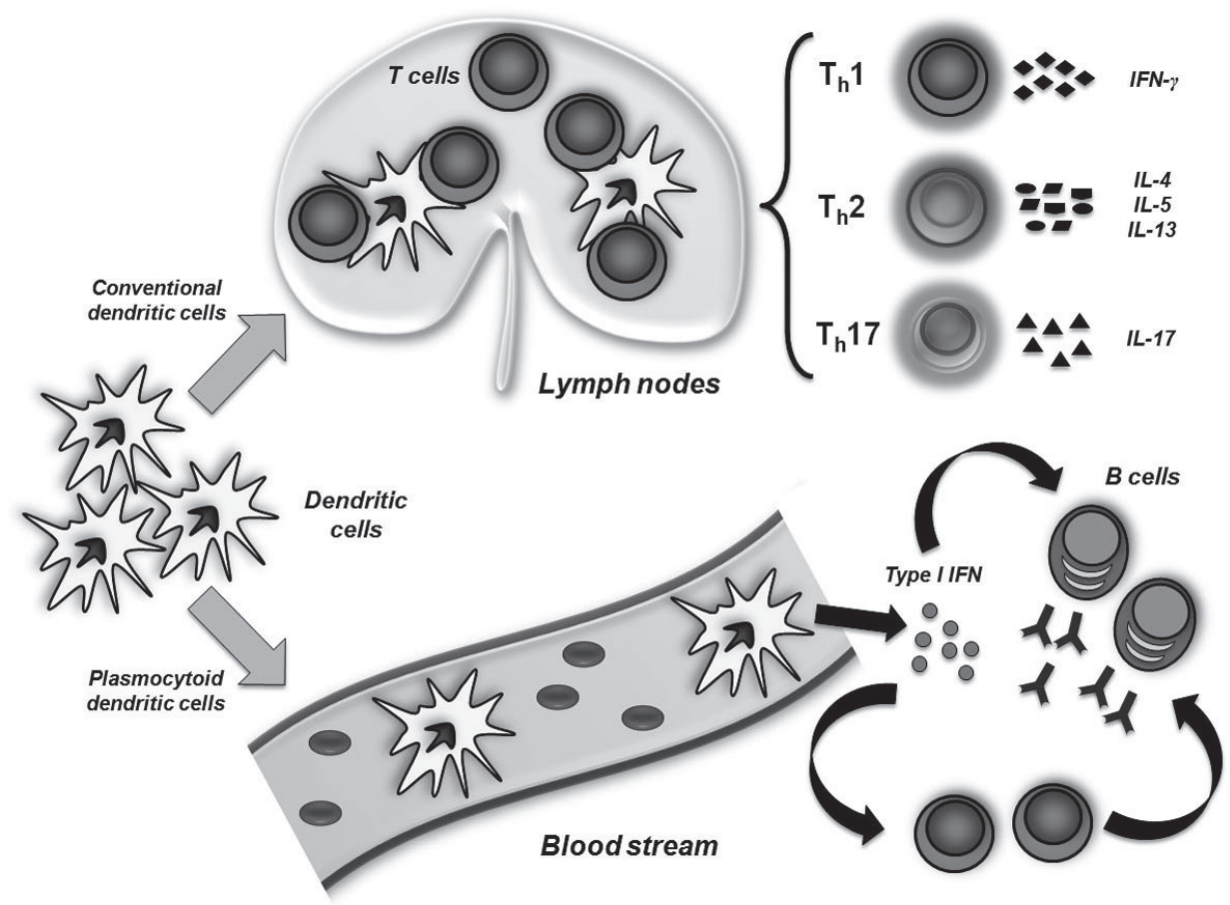

Figure 1. At least two different subsets of DC with distinctive functional properties can be found, conventional and plasmocytoid DCs. After CDCs capture antigens in peripheral tissues, they present them as peptide-MHC complexes and migrate to lymph nodes where they induce antigen-specific naïve $T$ cells to differentiate into effector $T$ cells. Depending on the molecular signals provided by cDCs during the immunological synapse, $T_{\text {helper }}$ cells can drive 3 different types of effector responses characterized by different cytokine profiles. $T_{h} 1$

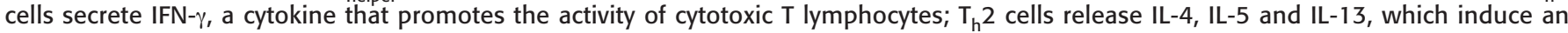
allergic-like immune response and $\mathrm{T}_{\mathrm{h}} 17$ cells secrete mainly IL-17 and participate in chronic inflammatory-autoimmune diseases, such as RA, MS (EAE) and SLE. Conversely, pDCs are CD11 c CD4+ cells that express TLR7 and 9, circulate in blood stream and secrete large amounts of type I IFN when activated. Through type-1 IFN secretion, pDCs can directly modulate adaptive immune responses by means of activating $T$ cells and inducing class switching on antigen-specific B cells. 
lineage that of $T_{h} 1$ and $T_{h} 2$ cells, but also can be antagonized by $T_{h} 1$ and $T_{h} 2$ cells and require Transporting Growth Factor (TGF)- $\beta$ for their differentiation (Weaver, Harrington et al. 2006). $T_{h}$ 17-polarized immune responses have been shown to be involved in the development of deleterious inflammation during EAE and RA in mice. This notion is supported by the observation that mice lacking IL-23 were resistant to EAE induced by sensitization with the myelin oligodendrocyte glycoprotein 35-55 (MOG) peptide (Cua, Sherlock et al. 2003). Similarly, the lack of IL-23 protected mice from suffering collagen-induced arthritis (CIA) (Murphy, Langrish et al. 2003).

In addition to their ability to define $\mathrm{T}$ cell subset differentiation and the nature of effector adaptive immunity, the complexity of DC function is underscored by the existence of at least 2 DC subsets, with distinctive functional properties: conventional and plasmocytoid DCs (cDCs and pDCs, respectively) (Figure 1). cDCs work as powerful APCs and efficient tissue sentinels that preferentially express TLR 3, 4, 5,6 and 8 , which results in the production of large amounts of TNF- $\alpha$, IL-1, IL-6 and IL-10 upon activation (Liu 2005; Diebold 2008). cDC can be further subdivided in different subsets depending on whether they reside in peripheral tissues, secondary lymphoid organs or circulate in blood stream, in addition circulating cDCs express different surface markers such as CD1a, CD16 and CD34 that allow even further differentiation (Ueno, Klechevsky et al. 2007). Conversely, pDCs can circulate in the blood stream and express low levels of MHC class II, CD86, undetectable levels of CD 80 and have a limited capacity to activate T lymphocytes (Liu 2005). Signaling through TLR7 and TLR9 induces pDC to release large amounts of type-1 IFN within the first 24 hours of stimulation (Liu 2005). It has been recently shown that as a result of type-1 IFN secretion, $\mathrm{pDCs}$ can directly contribute to the activation of $\mathrm{T}$ cells and induce class switching in antigen-specific $\mathrm{B}$ cells (Le Bon, Thompson et al. 2006). Subsequently, activated pDCs increase the expression of MHC class II molecules and costimulatory molecules CD80/CD86, secrete lower amounts of type- 1 IFN and become able to activate $\mathrm{CD}^{+} \mathrm{T}$ cells and induce them to produce IFN- $\gamma$ (Liu 2005). Along these lines, it is thought that during SLE, due to an increased frequency of apoptosis, self-antigens such as DNA and ribonucleoproteins become available and interact with TLRs on pDCs and promote the release of large amounts of type I IFN (Casciola-Rosen, Anhalt et al. 1994; Lovgren, Eloranta et al. 2004; Clancy, Neufing et al. 2006). Consistent with this notion, DCs from SLE patients show a mature phenotype characterized by an increased expression of co-stimulatory molecules and a decrease in the expression of inhibitory $\mathrm{F} c \gamma \mathrm{R}$ when compared to healthy controls. These phenotypic alterations can be critical for enhancing the immunogenicity of DCs and reducing their capacity to maintain $\mathrm{T}$ cell tolerance. Similar observations have been made in mouse models for SLE, which show DCs with increased capacity to prime naïve $\mathrm{T}$ cells (Kalergis, Iruretagoyena et al. 2009).

DCs can also shape the fate of an adaptive immune response depending on their maturation state when they interact with T cells. As mentioned above, mature DCs express high levels of MHC class II and co-stimulatory molecules. In contrast, immature DCs, in general, express low levels of MHC class II and co-stimulatory molecules and secrete low levels of pro-inflammatory cytokines. The interaction between immature DCs and T cells in peripheral lymphoid organs is considered to induce antigen-specific unresponsiveness by $\mathrm{T}$ cells due to anergy or deletion (Steinman, Hawiger et al. 2003; Diebold 2008). Evidence for this dual function of DCs was provided by using double transgenic mice expressing inducible Cre recombinase in $\mathrm{CD}_{11 \mathrm{c}^{+}}$cells propst lapnel et al. 2003. In this system, Cre activity was induced by the injection of Tamoxifen and resulted in the presentation of transgenic CTL epitopes by DCs. When tamoxifen was supplied, together with an agonistic anti-CD40 antibody, CTL epitopes were presented by activated DC, and when tamoxifen was injected alone, it led to the presentation of CTL epitopes by resting DCs. While activated DCs were able to prime naïve specific $C D 8^{+} \mathrm{T}$ cells, antigen presentation by resting DCs resulted in tolerance of specific $\mathrm{CD}^{+} \mathrm{T}$ cells (Probst, Lagnel et al. 2003). Recent work suggests that tolerance depends on intrinsic CD8 ${ }^{+} \mathrm{T}$ cell mechanisms involving the co-stimulatory molecules PD-1 and CTLA-4 (Probst, McCoy et al. 2005).

In addition, subsets of DCs have the ability to induce peripheral $\mathrm{T}$ cell tolerance by $\mathrm{T}$-cell extrinsic mechanisms. It has been demonstrated that in the gut, $\mathrm{CD} 103^{+} \mathrm{DCs}$ are able to induce the development of Foxp $3^{+} \mathrm{T}_{\text {regs }}$ in a TGF- $\beta$ and retinoic acid dependent manner, the same results were observed in DCs from the intestinal lamina propria (Coombes, Siddiqui et al. 2007; Sun, Hall et al. 2007). Furthermore, it has been shown in mice that in the periphery $\mathrm{CD} 8{ }^{+} \mathrm{CD} 205^{+}$, DCs induce conversion of naïve CD4 $4^{+}$T cells into $T_{\text {regs }}$ by means of TGF- $\beta$ secretion and programmed death 1 ligand (PD-L1) pathway (Wang, Pino-Lagos et al. 2008; Yamazaki, Dudziak et al. 2008).

Immunological synapse: a macromolecular machine controlling $T$ cell immunity and tolerance

During antigen recognition, $\mathrm{T}$ cells and APCs need to be in close contact to allow the interaction between the TCR and the cognate pMHC ligands (Gonzalez, Prado et al. 2008). This interplay takes place across the immunological synapse (IS), a complex and specialized supramolecular junction at the contact interface between the $\mathrm{T}$ cell and the pMHC-loaded APC (Dustin, Olszowy et al. 1998; Grakoui, Bromley et al. 1999). The most significant advances in this field were made when technical approaches consisting of lipid bilayers and deconvolution microscopy became available (Dustin 2008). This approach allowed the establishment of a model in which synapses are constituted as a large central cluster of TCRs and PKC- $\theta$ known as central supramolecular activation cluster (cSMAC) surrounded by a ring of LFA-1/ICAM-1 pairs, known as peripheral supramolecular activation clusters (pSMAC) (Monks, Freiberg et al. 1998; Davis and Dustin 2004). Later studies revealed a third zone at the synapse, the distal SMAC (dSMAC), containing significant amounts of CD45 molecules (Dustin 2008). When the concept of IS was initially introduced, it implied adhesion, polarization and stability (Dustin and Colman 2002; Dustin 2008). However, the interaction of $\mathrm{T}$ cells with DCs involves several distinctive features that obliged broadening the implications of the IS. Synapses between DCs and T cells are characterized by rapid and brief serial DC-T cell contacts in the early stages of $\mathrm{T}$ cell interaction and only by the conformation of a stable DC-T cell interaction in later stages (Dustin, Tseng et al. 2006; Bousso 2008). The first stage can occur with a small amount of pMHC present per DC (Henrickson, Mempel et al. 2008). It seems that $\mathrm{T}$ cells first scan for different antigen amounts 
on the DC surface until they finally find one with the pMHC density required for establishing a formal-stable synapse. These "mobile junctions" have been called kinapses (Dustin 2008; Dustin 2009). This process leads to T cell signaling only based on motile interactions with the APCs that in vivo result in tolerance. During this phase, $\mathrm{T}$ cells are able to integrate critical signals through the TCR and it is possible that the balance between synapse and kinapse modes during $\mathrm{T}$ cell priming may alter the differentiation of these cells during the $\mathrm{T}$ cell response (Dustin 2008). In addition, DC-T cell synapses can be stabilized by co-stimulatory molecules on the surface of DCs and cytokines secreted by these cells in response to PAMP stimulation (Figure 2). Therefore, environments enriched in inflammatory signals would favor the formation of stableactivating synapses over kinapses that could contribute to the activation of $\mathrm{T}$ cells upon encountering low avidity ligands, such as self-pMHC (Iruretagoyena, Wiesendanger et al. 2006). This process is likely to favor the onset of an autoimmune response in susceptible individuals (Figure 2).

\section{T cell activation in effector immunological synapses}

TCR recognition by a cognate $\mathrm{pMHC}$ ligand leads to $\mathrm{T}$ cell activation with subsequent $\mathrm{T}$ cell proliferation and differentiation (Carreno, Gonzalez et al. 2006; Iruretagoyena, Wiesendanger et al. 2006). After TCR engagement, the Srcfamily tyrosine-kinases lck and fyn are recruited to the cell membrane to catalyze the phosphorilation of CD3 $\xi$ molecules in the tyrosine-based activating motifs (ITAM) (Gonzalez, Carreno et al. 2007). However, as mentioned before, stimulation via the TCR is not sufficient to induce a productive $\mathrm{T}$ response, and instead might induce anergy on naive $\mathrm{T}$ cells. Co-stimulatory signals are crucial to optimize $\mathrm{T}$ cell activation. The CD28-CD80/CD86 interaction is the classical co- stimulatory pathway and its activation is known to enhance initial Src kinases induction (Goronzy and Weyand 2008). In addition, in opposition to the activation process, there are strong counter-regulating mechanisms for $\mathrm{T}$ cell activation, which are provided by inhibitory signaling pathways. A very proficient one is the T-cell-expressed molecule cytotoxic T-lymphocyte antigen-4 (CTLA-4), which binds the same ligands as CD28, but with higher affinity (Goronzy and Weyand 2008; Sharpe 2009). Of note, CTLA4 binds to B7.1 rather than B7.2, and this binding leads to the preferential relocalization of CTLA-4 in the IS. A key molecule in the signaling pathways involved in $\mathrm{T}$ cell activation is PKC- $\theta$. The crucial role of PKC- $\theta$ in mediating TCR dependent $\mathrm{T}$ cell activation in vivo has been shown by two different groups using PKC- $\theta-/-$ mice (Sun, Arendt et al. 2000; Pfeifhofer, Kofler et al. 2003). The two works have independently demonstrated that $\mathrm{T}$ cell proliferation and IL-2 production dramatically decreased in mutant mice upon CD3/CD28 stimulation. However, later experiments, in which PKC- $\theta$ deficient mice were immunized with lymphocytic choriomeningitis virus (LCMV), showed that the ability to induce an immune response against LCMV was not impaired in mutant mice (Berg-Brown, Gronski et al. 2004) Conversely, studies performed with EAE mice indicate that PKC- $\theta$ is crucial in antigen-specific Th1 and Th17 responses (SalekArdakani, So et al. 2005; Tan, Zhao et al. 2006), suggesting a differential requirement of PKC- $\theta$ signaling in effector $\mathrm{T}$ cell subsets with a necessary function in autoimmune related pathways.

\section{Regulatory $T$ cells as controllers of effector $T$ cells responses}

In the last few years, several subsets of regulatory $\mathrm{T}$ cells ( $\mathrm{T}$ regs) capable of controlling effector $\mathrm{T}$ cells responses have been described, but they can be divided into at least two types: natural $\mathrm{T}$ regs and induced or adaptive $\mathrm{T}$ regs (Brusko,

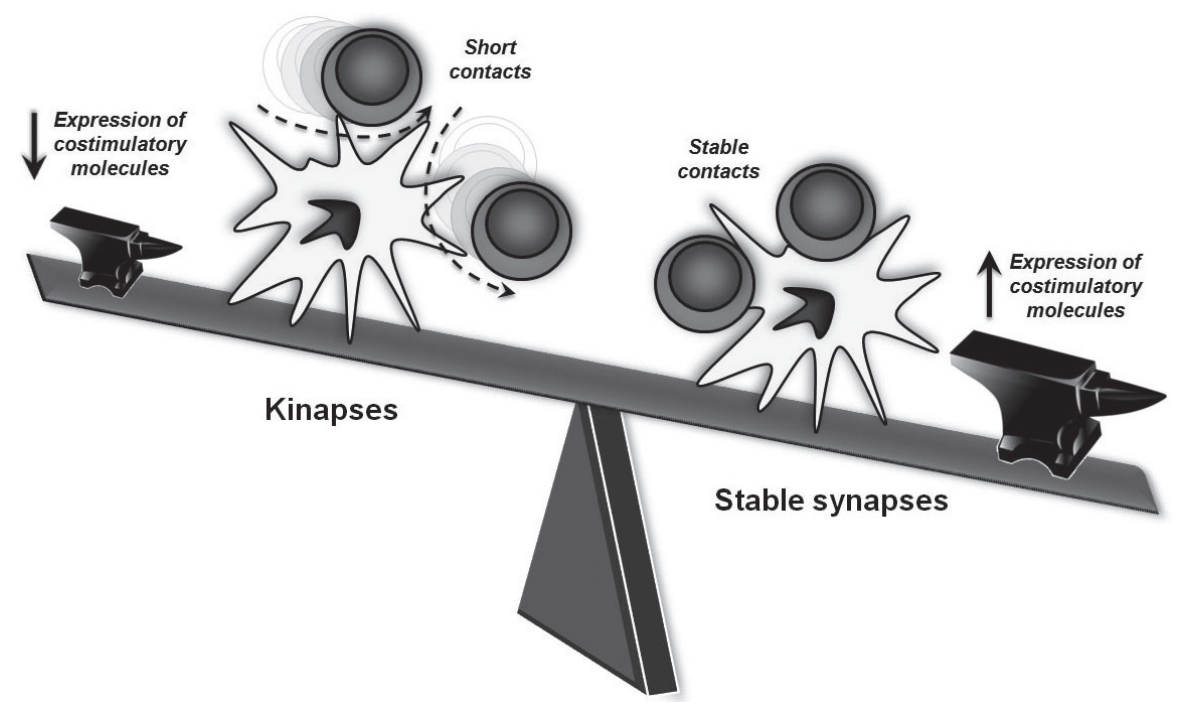

Figure 2. In early stages of DC-T cell interactions, DC-T cell contacts are short and non lasting, which allows T cells to scan for cognate peptide-MHC complexes on the DC surface. These unstable-mobile junctions have been denominated "Kinapses" and although they can lead to TCR signaling, kinapses have been associated with lack of immunity an induction of tolerance in vivo. In response to pathogen-associated molecular patterns DCs express co-stimulatory molecules that shift the balance towards the establishment of immunological synapses, which halt T cell migration and allow them to dwell with DCs for longer periods of time, facilitating sustained TCR-signaling on T cells. 
Putnam et al. 2008). They are both CD25+ and Foxp3+. While natural $\mathrm{T}$ regs are spontaneously generated in the thymus, induced $\mathrm{T}$ regs are derived from peripheral $\mathrm{CD}^{2} 5^{+} \mathrm{Foxp} 3^{-}$ $\mathrm{CD}^{+} \mathrm{T}$ cells after stimulation with transforming growth factor (TGF)- $\beta$ (Brusko, Putnam et al. 2008) (Figure 3). Recent evidence suggests that, in addition to their effect on activated $\mathrm{CD}^{+} \mathrm{T}$ cells, they can also influence $\mathrm{CD} 8^{+}$responses and $\mathrm{B}$ cell function (Lim, Hillsamer et al. 2005). During an effective immune response against pathogens, the function of effector cells is confined by $\mathrm{T}$ regs in order to ensure tolerance to self-antigens. Alternatively, in the context of autoimmunity, the $\mathrm{T}$ reg function is ineffective. We could suppose that this is due to either defective $\mathrm{T}$ reg cells or the $\mathrm{T}$ reg pool being overwhelmed by autorreactive effector $\mathrm{T}$ cells (Figure 3 ). The first hypothesis does not seem to be a relevant mechanism in the pathogenesis of autoimmune states since in classic autoimmune diseases such as type 1 Diabetes and Multiple Sclerosis (MS) the frequency of T regs is not altered (Brusko, Putnam et al. 2008). Similarly, although studies done with SLE patients show a lower number of $\mathrm{T}$ regs in peripheral blood, the total number of $\mathrm{CD} 4^{+} \mathrm{Foxp}^{+}$cells is not reduced in older mice that develop lupus (Horwitz 2008). Tregs regulate adaptive immune responses by several mechanisms that converge in the generation of a regulatory milieu at the site of antigen presentation and that promote the suppression of APC functions and/or T cell activation. Of note, experiments including adoptive transfer of Tregs populations show that their regulatory effect persists even after the transferred cells are removed. Data addressing $\mathrm{T}$ reg suppressive activity in MS suggest that $\mathrm{CD}^{+}{ }^{+}$oxp $^{+}$cells show decreased function
(Brusko, Putnam et al. 2008). Furthermore, experiments performed in vivo show that $\mathrm{T}$ regs from $\mathrm{C} 57 \mathrm{BL} / 6$ mice reduced the severity of EAE in vivo (Vandenbark and Offner 2008). Data on type 1 Diabetes are contradictory, and while in the case of murine lupus, several groups have reported that the $\mathrm{T}$ reg suppressive function is not altered, the initial approach was the existence of a deficient $\mathrm{T}$ reg repertory that allows the expansion of a protective immune response (Brusko, Putnam et al. 2008). Conversely, emerging data suggest that resistance of effector $\mathrm{T}$ cells to $\mathrm{T}$ reg supression may be a relevant mechanism to break tolerance. Experiments performed with lupus MRL/Mp mice show that $\mathrm{CD} 4^{+} \mathrm{CD} 25^{-} \mathrm{T}$ cells have reduced sensitivity to suppression (Monk, Spachidou et al. 2005). Similar results have been seen in human SLE (Venigalla, Tretter et al. 2008)

Pharmacological modulation of DC function as a therapeutic approach to promote tolerance

The majority of autoimmune diseases are treated with immunosuppressive drugs that act most of the time in a non-specific fashion, hampering the whole system, as is the case with glucocorticoids. The use of these medications is frequently associated with infectious complications, which carry substantial morbidity and sometimes mortality. Consequently, the development of antigen-specific therapies is warranted. DCs are good candidates to either treat or prevent autoimmune diseases due to their natural ability to induce tolerance in vivo. As mentioned before, besides $\mathrm{T}$ reg generation, $\mathrm{DCs}$ can induce peripheral tolerance by

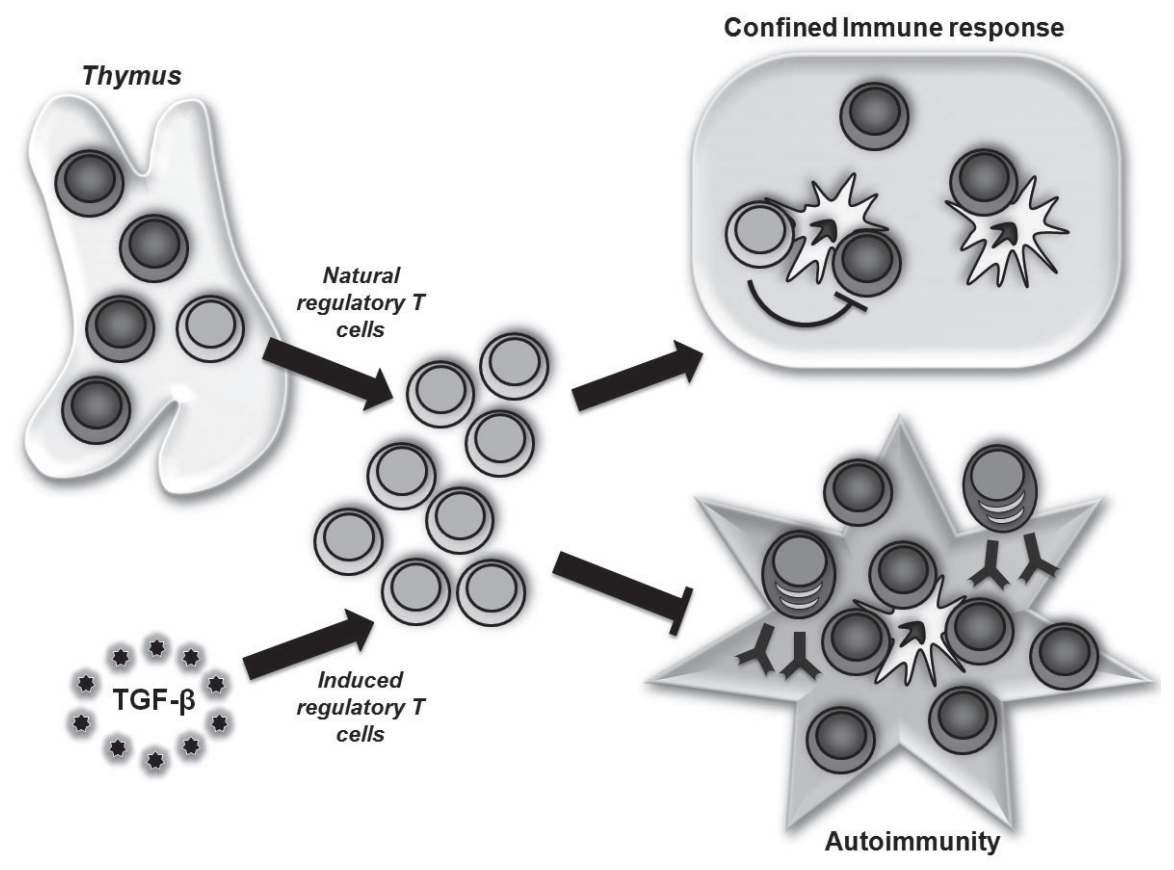

Figure 3. In the thymus, high affinity TCR-self-pMHC interactions promote the generation and expansion of natural regulatory $\mathrm{T}_{\text {cells }}\left(\mathrm{T}_{\text {regs }}\right)$ that are released into circulation. In addition, in the periphery CD4 ${ }^{+}$Foxp $3^{+} \mathrm{T}_{\text {regs }}$ are induced in a TGF- -dependent manner. During adaptive immune responses against pathogens, both subsets of $\mathrm{T}$ regs confine inflammation in order to avoid damage to self-tissues. During autoimmune processes it is thought that $\mathrm{T}_{\text {regs }}$ fail to limit inflammatory damage because they are either defective or because their target cells are resistant to suppression, leading to uncontrolled inflammation and destruction of host tissues. 
either rendering auto reactive $\mathrm{T}$ cell anergic or by deleting them. Immature or tolerogenic DCs express low quantities of surface MHC molecules, have a low ratio of co-stimulatory to inhibitory signals and an impaired ability to produce proinflammatory cytokines. Furthermore, DCs can be loaded with a wide range of antigens adding specificity to their potential. However, one of the problems with autoimmune disease therapy with immature DCs is the potential loss of their immature phenotype when confronted with a proinflammatory environment. Noteworthy, DC maturation can be pharmacologically regulated in vitro in order to obtain cells resistant to the danger signals present in the receiver. In an effort to generate in vivo tolerogenic DCs, DCs propagated in vitro have been manipulated with several anti-inflammatory and immunosuppressive drugs, including vitamin-D3, aspirin, rapamycin and adragrapholide, that interfere with various checkpoints of DC differentiation and expansion (Hackstein and Thomson 2004; Iruretagoyena, Tobar et al. 2005). Several lines of evidence show that the active metabolite of vitamin-D3, 1,25-dihydroxyvitamin D3 (calcitriol) blocks the differentiation of human-derived DCs and promotes the expression of monocyte marker CD14 in vitro (Berer, Stockl et al. 2000; Hackstein and Thomson 2004). Although mice knocked out for vitamin D receptor do not present a significantly lower number of CD11+ DC compared to the wild types, t-1D animal studies using NOD mice have demonstrated that treatment with calcitriol can inhibit the development of diabetes, which suggests a relevant effect of calcitriol in vivo (Gregori, Giarratana et al. 2002; Hackstein and Thomson 2004). Surprisingly, aspirin (acetylsalicylate) has been shown in a dose dependent manner to inhibit the expression of CD40, CD80, CD86 and MHC II molecules of bone-marrow derived DCs and to enhance their endocytic capacity (Hackstein, Morelli et al. 2001; Hackstein and Thomson 2004). In the case of rapamycin, a bacterial macrolide antibiotic with potent immunosuppressive action, there is in vitro and in vivo evidence showing that this drug is able to block DC maturation and Flt3L-induced expansion (Hackstein, Taner et al. 2003). Likewise, our group provided evidence showing that andragrapholide treated DCs exhibited an immature phenotype and were unable to activate specific $\mathrm{T}$ cells as indicated by IL-2 production. Noteworthy, when andragrapholide was administered to mice induced to develop EAE by injection of MOG, they developed a milder disease (Iruretagoyena, Tobar et al. 2005). Emerging therapies for RA exploit the tolerogenic capacity of DCs. Experimental evidence shows that pDCs, when activated via TLRs or following engagement of CD80 or CD86 with CTLA-4, express indoleamine 2,3-dioxygenase (IDO) and induce $\mathrm{T}$ reg cells. Furthermore, induction of IDO in animal models of arthritis controls the accumulation of pathogenic $T$ cells at the site of inflammation and DCs genetically modified to express IDO can reverse the development of collagen induced arthritis (Bianco, Kim et al. 2009; Criado, Simelyte et al. 2009). Although this area has great potential because it is antigen specific and does not suppress the whole immune system, it is only applicable to $\mathrm{AD}$ caused by known self-antigens. Thus, research efforts should also focus on identifying the relevant antigens involved in autoimmunity, which will have a significant impact in the design of new and safer therapies.

\section{REFERENCES}

BANCHEREAU, J. and R. M. STEINMAN (1998). "Dendritic cells and the control of immunity." Nature 392(6673): 245-52.

BERER, A., J. STOCKL, et al. (2000). "1,25-Dihydroxyvitamin D(3) inhibits dendritic cell differentiation and maturation in vitro." Exp Hematol 28(5): 575-83.

BERG-BROWN, N. N., M. A. GRONSKI, et al. (2004). "PKCtheta signals activation versus tolerance in vivo." J Exp Med 199(6): 743-52.

BETTINI, M. L. and D. A. VIGNALI "Development of thymically derived natural regulatory T cells." Ann N Y Acad Sci 1183: 1-12.

BIANCO, N. R., S. H. KIM, et al. (2009). "Therapeutic effect of exosomes from indoleamine 2,3-dioxygenase-positive dendritic cells in collageninduced arthritis and delayed-type hypersensitivity disease models." Arthritis Rheum 60(2): 380-9.

BOUSSO, P. (2008). "T-cell activation by dendritic cells in the lymph node: lessons from the movies." Nat Rev Immunol 8(9): 675-84.

BRESSON, D. and M. VON HERRATH (2007). “Moving towards efficient therapies in type 1 diabetes: to combine or not to combine?" Autoimmun Rev 6(5): 315-22.

BRIMNES, M. K., L. BONIFAZ, et al. (2003). “Influenza virus-induced dendritic cell maturation is associated with the induction of strong $\mathrm{T}$ cell immunity to a coadministered, normally nonimmunogenic protein." J Exp Med 198(1): 133-44.

BRUSKO, T. M., A. L. PUTNAM, et al. (2008). “Human regulatory T cells: role in autoimmune disease and therapeutic opportunities." Immunol Rev 223: 371-90.

CARREÑO, L. J., P. A. GONZALEZ, et al. (2006). "Modulation of T cell function by TCR/pMHC binding kinetics." Immunobiology 211(1-2): 47-64.

CASCIOLA-ROSEN, L. A., G. ANHALT, et al. (1994). "Autoantigens targeted in systemic lupus erythematosus are clustered in two populations of surface structures on apoptotic keratinocytes." J Exp Med 179(4): 1317-30.

CHOY, E. H. and G. S. PANAYI (2001). "Cytokine pathways and joint inflammation in rheumatoid arthritis." N Engl J Med 344(12): 907-16.

CLANCY, R. M., P. J. NEUFING, et al. (2006). "Impaired clearance of apoptotic cardiocytes is linked to anti-SSA/Ro and -SSB/La antibodies in the pathogenesis of congenital heart block." J Clin Invest 116(9): 2413-22.

COFFMAN, R. L. (2006). "Origins of the T(H)1-T(H)2 model: a personal perspective." Nat Immunol 7(6): 539-41.

COOMBES, J. L., K. R. SIDDIQUI, et al. (2007). “A functionally specialized population of mucosal CD103+ DCs induces Foxp3+ regulatory T cells via a TGF-beta and retinoic acid-dependent mechanism." J Exp Med 204(8): 1757-64.

CRIADO, G., E. SIMELYTE, et al. (2009). “Indoleamine 2,3 dioxygenasemediated tryptophan catabolism regulates accumulation of Th1/Th17 cells in the joint in collagen-induced arthritis." Arthritis Rheum 60(5): 1342-51.

CUA, D. J., J. SHERLOCK, et al. (2003). "Interleukin-23 rather than interleukin-12 is the critical cytokine for autoimmune inflammation of the brain." Nature 421(6924): 744-8.

DALL'ERA, M. and D. WOFSY (2009). "Systemic lupus erythematosus clinical trials-an interim analysis." Nat Rev Rheumatol 5(6): 348-51.

DAVIS, D. M. and M. L. DUSTIN (2004). "What is the importance of the immunological synapse?" Trends Immunol 25(6): 323-7.

DEANGELIS, T. and F. LUBLIN (2008). "Multiple sclerosis: new treatment trials and emerging therapeutic targets." Curr Opin Neurol 21(3): 26171.

DIEBOLD, S. S. (2008). "Determination of T-cell fate by dendritic cells." Immunol Cell Biol 86(5): 389-97.

DUSTIN, M. L. (2008). "Hunter to gatherer and back: immunological synapses and kinapses as variations on the theme of amoeboid locomotion." Annu Rev Cell Dev Biol 24: 577-96.

DUSTIN, M. L. (2008). "T-cell activation through immunological synapses and kinapses." Immunol Rev 221: 77-89.

DUSTIN, M. L. (2009). “The cellular context of T cell signaling." Immunity 30(4): 482-92.

DUSTIN, M. L. and D. R. COLMAN (2002). “Neural and immunological synaptic relations." Science 298(5594): 785-9.

DUSTIN, M. L., M. W. OLSZOWY, et al. (1998). “A novel adaptor protein orchestrates receptor patterning and cytoskeletal polarity in T-cell contacts." Cell 94(5): 667-77.

DUSTIN, M. L., S. Y. TSENG, et al. (2006). "T cell-dendritic cell immunological synapses." Curr Opin Immunol 18(4): 512-6. 
EISENBERG, R. (2009). "Why can't we find a new treatment for SLE?" J Autoimmun 32(3-4): 223-30.

EIZIRIK, D. L., M. L. COLLI, et al. (2009). "The role of inflammation in insulitis and beta-cell loss in type 1 diabetes." Nat Rev Endocrinol 5(4): 219-26.

GALLEGOS, A. M. and M. J. BEVAN (2004). "Central tolerance to tissuespecific antigens mediated by direct and indirect antigen presentation." J Exp Med 200(8): 1039-49.

GARDNER, J. M., J. J. DEVOSS, et al. (2008). “Deletional tolerance mediated by extrathymic Aire-expressing cells." Science 321(5890): 843-7.

GONZALEZ, P. A., L. J. CARREÑO, et al. (2007). "Modulation of immunological synapse by membrane-bound and soluble ligands." Cytokine Growth Factor Rev 18(1-2): 19-31.

GONZALEZ, P. A., C. E. PRADO, et al. (2008). “Respiratory syncytial virus impairs $T$ cell activation by preventing synapse assembly with dendritic cells." Proc Natl Acad Sci U S A 105(39): 14999-5004.

GORONZY, J. J. and C. M. WEYAND (2008). “T-cell co-stimulatory pathways in autoimmunity." Arthritis Res Ther 10 Suppl 1: S3.

GRAKOUI, A., S. K. BROMLEY, et al. (1999). "The immunological synapse: a molecular machine controlling T cell activation." Science 285(5425): 221-7.

GREGORI, S., N. GIARRATANA, et al. (2002). “A 1alpha,25dihydroxyvitamin $\mathrm{D}(3)$ analog enhances regulatory T-cells and arrests autoimmune diabetes in NOD mice." Diabetes 51(5): 1367-74

HACKSTEIN, H., A. E. MORELLI, et al. (2001). "Aspirin inhibits in vitro maturation and in vivo immunostimulatory function of murine myeloid dendritic cells." J Immunol 166(12): 7053-62.

HACKSTEIN, H., T. TANER, et al. (2003). "Rapamycin inhibits IL-4--induced dendritic cell maturation in vitro and dendritic cell mobilization and function in vivo." Blood 101(11): 4457-63.

HACKSTEIN, H. and A. W. THOMSON (2004). “Dendritic cells: emerging pharmacological targets of immunosuppressive drugs." Nat Rev Immunol 4(1): 24-34.

HENRICKSON, S. E., T. R. MEMPEL, et al. (2008). “T cell sensing of antigen dose governs interactive behavior with dendritic cells and sets a threshold for T cell activation." Nat Immunol 9(3): 282-91.

HERRADA, A. A., F. J. CONTRERAS, et al. "Aldosterone promotes autoimmune damage by enhancing Th17-mediated immunity." J Immunol 184(1): 191-202

HERRADA, A. A., F. J. CONTRERAS, et al. (2007). “Immune complexinduced enhancement of bacterial antigen presentation requires Fcgamma receptor III expression on dendritic cells." Proc Natl Acad Sci U S A 104(33): 13402-7.

HORWITZ, D. A. (2008). "Regulatory T cells in systemic lupus erythematosus: past, present and future." Arthritis Res Ther 10(6): 227.

IRURETAGOYENA, M. I., C. A. RIEDEL, et al. (2008). "Activating and inhibitory Fcgamma receptors can differentially modulate $\mathrm{T}$ cellmediated autoimmunity." Eur J Immunol 38(8): 2241-50.

IRURETAGOYENA, M. I., J. A. TOBAR, et al. (2005). “Andrographolide interferes with $\mathrm{T}$ cell activation and reduces experimental autoimmune encephalomyelitis in the mouse." J Pharmacol Exp Ther 312(1): 366-72.

IRURETAGOYENA, M. I., M. WIESENDANGER, et al. (2006). "The dendritic cell-T cell synapse as a determinant of autoimmune pathogenesis." Curr Pharm Des 12(2): 131-47.

KALERGIS, A. M. (2003). "Modulation of T cell immunity by TCR/pMHC dwell time and activating/inhibitory receptor pairs on the antigenpresenting cell." Curr Pharm Des 9(3): 233-44.

KALERGIS, A. M., M. I. IRURETAGOYENA, et al. (2009). "Modulation of nuclear factor-kappaB activity can influence the susceptibility to systemic lupus erythematosus." Immunology 128(1 Suppl): e306-14.

Kalergis, A. M. and J. V. Ravetch (2002). "Inducing tumor immunity through the selective engagement of activating Fcgamma receptors on dendritic cells." J Exp Med 195(12): 1653-9.

KRAKOWSKI, M. and T. OWENS (1996). "Interferon-gamma confers resistance to experimental allergic encephalomyelitis." Eur J Immuno 26(7): 1641-6.

LANZAVECCHIA, A. and F. SALLUSTO (2001). "Regulation of T cell immunity by dendritic cells." Cell 106(3): 263-6.

LE BON, A., C. THOMPSON, et al. (2006). "Cutting edge: enhancement of antibody responses through direct stimulation of $\mathrm{B}$ and $\mathrm{T}$ cells by type $\mathrm{I}$ IFN." J Immunol 176(4): 2074-8.

LEANDRO, M. J., J. C. EDWARDS, et al. (2002). "An open study of B lymphocyte depletion in systemic lupus erythematosus." Arthritis Rheum 46(10): 2673-7.

LIM, H. W., P. HILLSAMER, et al. (2005). “Cutting edge: direct suppression of B cells by CD4+ CD25+ regulatory T cells." J Immunol 175(7): 4180-3.
LIU, Y. J. (2005). "IPC: professional type 1 interferon-producing cells and plasmacytoid dendritic cell precursors." Annu Rev Immunol 23: 275-306.

LOVGREN, T, M. L. ELORANTA, et al. (2004). “Induction of interferonalpha production in plasmacytoid dendritic cells by immune complexes containing nucleic acid released by necrotic or late apoptotic cells and lupus IgG." Arthritis Rheum 50(6): 1861-72.

LUTZ, M. B. and C. KURTS (2009). "Induction of peripheral CD4+ T-cell tolerance and CD8+ T-cell cross-tolerance by dendritic cells." Eur J Immunol 39(9): 2325-30

MCCAUGHTRY, T. M., T. A. BALDWIN, et al. (2008). "Clonal deletion of thymocytes can occur in the cortex with no involvement of the medulla." J Exp Med 205(11): 2575-84.

MERRILL, J. T., C. M. NEUWELT, et al. "Efficacy and safety of rituximab in moderately-to-severely active systemic lupus erythematosus: the randomized, double-blind, phase II/III systemic lupus erythematosus evaluation of rituximab trial." Arthritis Rheum 62(1): 222-33.

MONK, C. R., M. SPACHIDOU, et al. (2005). “MRL/Mp CD4+,CD25- T cells show reduced sensitivity to suppression by $\mathrm{CD} 4+, \mathrm{CD} 25+$ regulatory $\mathrm{T}$ cells in vitro: a novel defect of $\mathrm{T}$ cell regulation in systemic lupus erythematosus." Arthritis Rheum 52(4): 1180-4.

MONKS, C. R., B. A. FREIBERG, et al. (1998). "Three-dimensional segregation of supramolecular activation clusters in T cells." Nature 395(6697): 82-6.

MURPHY, C. A., C. L. LANGRISH, et al. (2003). “Divergent pro- and antiinflammatory roles for IL-23 and IL-12 in joint autoimmune inflammation." J Exp Med 198(12): 1951-7.

PETERSON, P., T. ORG, et al. (2008). “Transcriptional regulation by AIRE: molecular mechanisms of central tolerance." Nat Rev Immunol 8(12): 948-57.

PFEIFHOFER, C., K. KOFLER, et al. (2003). "Protein kinase C theta affects $\mathrm{Ca} 2+$ mobilization and NFAT cell activation in primary mouse T cells." J Exp Med 197(11): 1525-35.

PIRCHER, H., U. H. ROHRER, et al. (1991). "Lower receptor avidity required for thymic clonal deletion than for effector T-cell function." Nature 351(6326): 482-5.

PROBST, H. C., J. LAGNEL, et al. (2003). "Inducible transgenic mice reveal resting dendritic cells as potent inducers of CD8+ T cell tolerance." Immunity 18(5): 713-20.

PROBST, H. C., K. MCCOY, et al. (2005). "Resting dendritic cells induce peripheral CD8+ T cell tolerance through PD-1 and CTLA-4." Nat Immunol 6(3): 280-6.

RAHMAN, A. and D. A. ISENBERG (2008). "Systemic lupus erythematosus." N Engl J Med 358(9): 929-39.

SALEK-ARDAKANI, S., T. SO, et al. (2005). "Protein kinase Ctheta controls Th1 cells in experimental autoimmune encephalomyelitis." J Immunol 175(11): 7635-41.

SHARPE, A. H. (2009). “Mechanisms of costimulation.” Immunol Rev 229(1): 5-11.

SMITH, K. G., R. B. JONES, et al. (2006). “Long-term comparison of rituximab treatment for refractory systemic lupus erythematosus and vasculitis: Remission, relapse, and re-treatment." Arthritis Rheum 54(9): 2970-82.

STARR, T. K., S. C. JAMESON, et al. (2003). "Positive and negative selection of T cells." Annu Rev Immunol 21: 139-76.

STEINMAN, R. M., D. HAWIGER, et al. (2003). "Tolerogenic dendritic cells." Annu Rev Immunol 21: 685-711.

SUN, C. M., J. A. HALL, et al. (2007). "Small intestine lamina propria dendritic cells promote de novo generation of Foxp3 $\mathrm{T}$ reg cells via retinoic acid." J Exp Med 204(8): 1775-85.

SUN, Z., C. W. ARENDT, et al. (2000). "PKC-theta is required for TCRinduced NF-kappaB activation in mature but not immature $\mathrm{T}$ lymphocytes." Nature 404(6776): 402-7.

TAN, S. L., J. ZHAO, et al. (2006). "Resistance to experimental autoimmune encephalomyelitis and impaired IL-17 production in protein kinase C theta-deficient mice." J Immunol 176(5): 2872-9.

TAYLOR, P. C. and M. FELDMANN (2009). "Anti-TNF biologic agents: still the therapy of choice for rheumatoid arthritis." Nat Rev Rheumatol 5(10): 578-82

TOBAR, J. A., L. J. CARRENO, et al. (2006). "Virulent Salmonella enterica serovar typhimurium evades adaptive immunity by preventing dendritic cells from activating T cells." Infect Immun 74(11): 6438-48.

TOKUNAGA, M., K. SAITO, et al. (2007). "Efficacy of rituximab (anti-CD20) for refractory systemic lupus erythematosus involving the central nervous system." Ann Rheum Dis 66(4): 470-5.

UENO, H., E. KLECHEVSKY, et al. (2007). "Dendritic cell subsets in health and disease." Immunol Rev 219: 118-42. 
VANDENBARK, A. A. and H. OFFNER (2008). "Critical evaluation of regulatory $\mathrm{T}$ cells in autoimmunity: are the most potent regulatory specificities being ignored?" Immunology 125(1): 1-13.

VENIGALLA, R. K., T. TRETTER, et al. (2008). “Reduced CD4+,CD25- T cell sensitivity to the suppressive function of CD4+,CD25high,CD127 - / low regulatory T cells in patients with active systemic lupus erythematosus." Arthritis Rheum 58(7): 2120-30.

WALDRON-LYNCH, F. and K. C. HEROLD (2009). "Advances in Type 1 diabetes therapeutics: immunomodulation and beta-cell salvage." Endocrinol Metab Clin North Am 38(2): 303-17, viii.

WANG, L., K. PINO-LAGOS, et al. (2008). “Programmed death 1 ligand signaling regulates the generation of adaptive Foxp3+CD4+ regulatory T cells." Proc Natl Acad Sci U S A 105(27): 9331-6.

WEAVER, C. T., L. E. HARRINGTON, et al. (2006). “Th17: an effector CD4 T cell lineage with regulatory T cell ties." Immunity 24(6): 677-88.
WENINK, M. H., K. C. SANTEGOETS, et al. (2009). "The inhibitory FC gamma IIb receptor dampens TLR4-mediated immune responses and is selectively up-regulated on dendritic cells from rheumatoid arthritis patients with quiescent disease." J Immunol 183(7): 4509-20.

WILLENBORG, D. O., S. FORDHAM, et al. (1996). "IFN-gamma plays a critical down-regulatory role in the induction and effector phase of myelin oligodendrocyte glycoprotein-induced autoimmune encephalomyelitis." J Immunol 157(8): 3223-7.

WINGERCHUK, D. M., C. F. LUCCHINETTI, et al. (2001). “Multiple sclerosis: current pathophysiological concepts." Lab Invest 81(3): 263-81.

YAMAZAKI, S., D. DUDZIAK, et al. (2008). “CD8+ CD205+ splenic dendritic cells are specialized to induce Foxp3+ regulatory T cells." J Immunol 181(10): 6923-33.

YAMAZAKI, S., T. IYODA, et al. (2003). "Direct expansion of functional CD25+ CD4+ regulatory T cells by antigen-processing dendritic cells." J Exp Med 198(2): 235-47. 
